\title{
ON THE PRODUCT OF STABLE DIFFEOMORPHISMS
}

\author{
ATSURO SANNAMI
}

\begin{abstract}
It is proved that statements (a)-(c) are equivalent. (a) Structural ( $\Omega$-) stability conjecture. (b) If diffeomorphisms $f$ and $g$ are both structurally $(\Omega$-) stable, then so is $f \times g$. (c) If a diffeomorphism $f$ is structurally ( $\Omega-$ ) stable, then every diffeomorphism in a neighborhood of $f \times f^{-1}$ has only hyperbolic periodic points.
\end{abstract}

1. Introduction and statement of Theorem. Let $M$ be a compact smooth manifold without boundary and $\operatorname{Diff}^{1}(M)$ the space of $C^{1}$ diffeomorphisms on $M$ with the uniform $C^{1}$ topology. $f \in \operatorname{Diff}^{1}(M)$ is called structurally (resp. $\Omega$-) stable if there exists a neighborhood $\mathcal{U}$ of $f$ in $\operatorname{Diff}^{1}(M)$ such that for any $g \in \mathcal{Q}$, there is a homeomorphism $h: M \rightarrow M$ (resp. $h: \Omega(f) \rightarrow \Omega(g)$ ) with $g h=h f$ on $M$ (resp. on $\Omega(f)$ ), where $\Omega(f)$ denotes the nonwandering set of $f$.

The characterization of these stabilities is one of the basic problems in the theory of differentiable dynamical systems, and, on this subject, there are the well-known "stability conjectures".

Structural Stability Conjecture [6]. $f \in \operatorname{Diff}^{\prime}(M)$ is structurally stable if and only if $f$ satisfies Axiom A and the Strong transversality condition.

$\Omega$-Stability Conjecture $[5,13] . f \in \operatorname{Diff}^{1}(M)$ is $\Omega$-stable if and only if $f$ satisfies Axiom A and No cycle property.

For definitions, see above references and [4].

The sufficiency of Axiom A and the Strong transversality condition (resp. No cycle property) for structure (resp. $\Omega$-) stability has been proved $[9,11,13]$. Concerning the converses, in case of $\operatorname{dim} M=2$, they have been solved affirmatively $[1,12]$. But for $M$ with $\operatorname{dim} M \geqslant 3$, they are still open. In this paper, we will show that these conjectures are equivalent to the following natural questions, respectively. "Suppose $f, g \in \operatorname{Diff}^{1}(M)$ are both structurally (resp. $\Omega$-) stable. Then is $f \times g$ : $M \times M \rightarrow M \times M$ structurally (resp. $\Omega$-) stable, where $f \times g(x, y)=(f(x), g(y))$ for $(x, y) \in M \times M$ ?" For a precise statement of our result, we need a certain class of $C^{1}$ diffeomorphisms. We define

$$
F(M)=\operatorname{int}_{1}\left\{f \in \operatorname{Diff}^{1}(M): \text { all periodic points of } f \text { are hyperbolic }\right\},
$$

where int ${ }_{1}$ means interior with respect to the $C^{1}$ topology of $\operatorname{Diff}^{1}(M)$. Our result is

THEOREM. Statements (a)-(c) are equivalent.

(a) $f \in \operatorname{Diff}^{1}(M)$ is structurally (resp. $\Omega$-) stable if and only if $f$ satisfies Axiom A and the Strong transversality condition (resp. No cycle property).

Received by the editors May 20, 1982.

1980 Mathematics Subject Classification. Primary 58F10, 58F15. 
(b) If $f, g \in \operatorname{Diff}^{1}(M)$ are both structurally (resp. $\Omega$-) stable, then so is $f \times g$.

(c) If $f \in \operatorname{Diff}^{1}(M)$ is structurally (resp. $\Omega$-) stable, then $f \times f^{-1} \in F(M \times M)$.

Let $\operatorname{Per}(f)$ denote the set of all periodic points of $f \in \operatorname{Diff}^{1}(M)$. Since $p \in \operatorname{Per}(f)$ is hyperbolic for $f \in F(M)$, we can define

$$
\Lambda_{i}(f)=\operatorname{closure}\{p \in \operatorname{Per}(f): \text { stable dimension of } p=i\}
$$

for all integers $0 \leqslant i \leqslant \operatorname{dim} M$, where stable dimension means the dimension of the stable subspace of $T_{p} M$ corresponding to the hyperbolicity of $T f^{\pi(p)}: T_{p} M \rightarrow T_{p} M$ $(\pi(p)$ denotes the period of $p)$. The class $F(M)$ is closely related to the stabilities of diffeomorphisms $[1,3,8,12]$, and the essence of the proof of our Theorem is the following basic property of $F(M)$ proved by Mañé [2].

Lemma A. Let $f \in F(M)$ and $0<i<\operatorname{dim} M$. There exist constants $c>0,0<\lambda<$ 1 , and the unique Tf-invariant continuous splitting $T M \mid \Lambda_{i}(f)=E_{i}^{s}(f) \oplus E_{i}^{u}(f)$ such that

$$
\left\|T f^{n}\left|E_{i}^{s}(f)_{p}\|\cdot\| T f^{-n}\right| E_{i}^{u}(f)_{f^{n}(p)}\right\| \leqslant c \lambda^{n}
$$

for all $p \in \Lambda_{i}(f)$ and nonnegative integer $n$, where $E_{i}^{s}(f)_{p}, E_{i}^{u}(f)_{q}$ denote fibers over $p, q \in \Lambda_{i}(f)$.

In [7] Pliss proved the finiteness of the sinks and the sources for $f \in F(M)$, namely

Lemma B. Let $f \in F(M) . \Lambda_{0}(f)$ and $\Lambda_{\operatorname{dim} M}(f)$ are finite sets.

Note that by the theorem of Kupka and Smale (cf. [10]), it can be seen that if $f$ is $\Omega$-stable, then $f \in F(M)$.

2. Proof of Theorem. We prove only the case of " $\Omega$-stability". A similar argument gives the "structural stability" case.

(a) $\Rightarrow$ (b). Suppose $f, g \in \operatorname{Diff}^{1}(M)$ are both $\Omega$-stable. From (a), $f$ and $g$ satisfy Axiom $\mathrm{A}$ and No cycle property. Then $f \times g$ also satisfies Axiom A and No cycle property. We shall prove this fact in the next section. (If No cycle property is replaced by Strong transversality condition, then the proof is almost trivial.) From the $\Omega$-stability theorem [13], $f \times g$ is $\Omega$-stable.

(b) $\Rightarrow$ (c). If $f$ is $\Omega$-stable, then so is $f^{-1}$. From (b), $f \times f^{-1}$ is $\Omega$-stable. As is mentioned above, $\Omega$-stability of $f \times f^{-1} \in \operatorname{Diff}^{1}(M \times M)$ implies $f \times f^{-1} \in$ $F(M \times M)$.

Before proceeding to the proof of $(c) \Rightarrow(a)$, we need some basic properties of the product of diffeomorphisms in $F(M)$.

Let $g, h \in F(M)$ and $g \times h \in F(M \times M)$. Clearly,

$$
\operatorname{Per}(g \times h)=\operatorname{Per}(g) \times \operatorname{Per}(h)
$$

and

$$
\Lambda_{k}(g \times h)=\bigcup_{i+j=k} \Lambda_{i}(g) \times \Lambda_{j}(h) \text { for all } 0 \leqslant k \leqslant 2 \cdot \operatorname{dim} M
$$


From Lemmas A and $\mathrm{B}$, for all $0 \leqslant k \leqslant 2 \cdot \operatorname{dim} M$ there exists a $T(g \times h)$-invariant continuous splitting

$$
T(M \times M) \mid \Lambda_{k}(g \times h)=E_{k}^{s}(g \times h) \oplus E_{k}^{u}(g \times h)
$$

having the property in Lemma A. The uniqueness of the subbundles $E_{k}^{s}(g \times h)$, $E_{k}^{u}(g \times h)$ implies

$$
\begin{aligned}
& E_{k}^{s}(g \times h)=\bigcup_{i+j=k} E_{i}^{s}(g) \times E_{j}^{s}(h) \\
& E_{k}^{u}(g \times h)=\bigcup_{i+j=k} E_{i}^{u}(g) \times E_{j}^{u}(h)
\end{aligned}
$$

Now we shall prove (c) $\Rightarrow$ (a). This is the main part of the proof of our theorem. It is known that if the nonwandering set $\Omega(f)$ of $f \in F(M)$ is always a hyperbolic set, then (a) is established [3]. For $f \in F(M)$, Mañe showed that if $\Lambda_{i}(f)$ is a hyperbolic set for all $0 \leqslant i \leqslant \operatorname{dim} M$, then $\Omega(f)=\Lambda_{0}(f) \cup \cdots \cup \Lambda_{\operatorname{dim} M}(f)$, and this is a hyperbolic set [2]. Moreover, from Lemma $\mathrm{B}, \Lambda_{0}(f)$ and $\Lambda_{\operatorname{dim} M}(f)$ are both finite sets and, consequently, they are hyperbolic sets. Thus, we have only to show that if $f$ is $\Omega$-stable, then $\Lambda_{i}(f)$ is a hyperbolic set for all $0<i<\operatorname{dim} M$. In what follows, let $n=\operatorname{dim} M$ and we fix an $\Omega$-stable diffeomorphism $f$ and an integer $0<i<n$.

Since $f \in F(M)$, we have $f^{-1} \in F(M)$. Clearly for all $0 \leqslant i \leqslant n$,

$$
\begin{gathered}
\Lambda_{i}(f)=\Lambda_{n-i}\left(f^{-1}\right), \\
E_{i}^{s}(f)=E_{n-i}^{u}\left(f^{-1}\right), \quad E_{i}^{u}(f)=E_{n-i}^{s}\left(f^{-1}\right) .
\end{gathered}
$$

From hypothesis, $f \times f^{-1} \in F(M \times M)$. Hence, from Lemma $\mathrm{A}$, there exist constants $c>0$ and $0<\lambda<1$ such that

$$
\left\|T\left(f \times f^{-1}\right)^{m}\left|E_{n}^{s}\left(f \times f^{-1}\right)_{(p, q)}\|\cdot\| T\left(f \times f^{-1}\right)^{-m}\right| E_{n}^{u}\left(f \times f^{-1}\right)_{\left(f \times f^{-1}\right)^{m}(p, q)}\right\| \leqslant c \lambda^{m}
$$

for all $(p, q) \in \Lambda_{n}\left(f \times f^{-1}\right)$ and nonnegative integer $m$. From (2) and (4),

$$
\Lambda_{n}\left(f \times f^{-1}\right) \supset \Lambda_{i}(f) \times \Lambda_{n-i}\left(f^{-1}\right)=\Lambda_{i}(f) \times \Lambda_{i}(f) .
$$

From (3) and (5), for any $(x, y) \in \Lambda_{i}(f) \times \Lambda_{i}(f)$,

$$
E_{n}^{s}\left(f \times f^{-1}\right)_{(x, y)}=E_{i}^{s}(f)_{x} \times E_{i}^{u}(f)_{y}, \quad E_{n}^{u}\left(f \times f^{-1}\right)_{(x, y)}=E_{i}^{u}(f)_{x} \times E_{i}^{s}(f)_{y} .
$$

Since any riemannian metric on $M \times M$ is equivalent to a natural product of that on $M$, there is a constant $d>0$ such that, for any $(x, y) \in \Lambda_{n}\left(f \times f^{-1}\right)$,

$$
\begin{aligned}
& \left\|T\left(f \times f^{-1}\right)^{m}\left|E_{i}^{s}(f)_{x} \times E_{i}^{u}(f)_{y}\|\geqslant d \cdot\| T f^{m}\right| E_{i}^{s}(f)_{x}\right\|, \\
& \left\|T\left(f \times f^{-1}\right)^{-m}\left|E_{i}^{u}(f)_{x} \times E_{i}^{s}(f)_{y}\|\geqslant d \cdot\| T\left(f^{-1}\right)^{-m}\right| E_{i}^{s}(f)_{y}\right\| .
\end{aligned}
$$

Putting $(p, q)=\left(x, f^{m}(x)\right)$ in (6) for $x \in \Lambda_{i}(f)$, we have from (7) and (8),

$$
c \lambda^{m} \geqslant d^{2} \cdot\left\|T f^{m}\left|E_{i}^{s}(f)_{x}\|\cdot\| T f^{m}\right| E_{i}^{s}(f)_{x}\right\|=d^{2} \cdot\left\|T f^{m} \mid E_{i}^{s}(f)_{x}\right\|^{2} .
$$


Therefore we get

$$
\left\|T f^{m} \mid E_{i}^{s}(f)_{x}\right\| \leqslant d^{-1} \cdot \sqrt{c} \cdot(\sqrt{\lambda})^{m}
$$

for all $x \in \Lambda_{i}(f)$ and nonnegative integer $m$. Similarly,

$$
\left\|T f^{-m} \mid E_{i}^{u}(f)_{x}\right\| \leqslant d^{\prime-1} \cdot \sqrt{c} \cdot(\sqrt{\lambda})^{m}
$$

for some constant $d^{\prime}>0$ and for all $x \in \Lambda_{i}(f)$ and $m \geqslant 0$. This means that $\Lambda_{i}(f)$ is a hyperbolic set.

3. The product of diffeomorphisms satisfying Axiom $A$ and No cycle property. In this section we prove the following Proposition used in the proof of our Theorem.

Proposition. If $f$ and $g$ satisfy Axiom $\mathrm{A}$ and No cycle property, then so does $f \times g$.

The essence of the proof is

Lemma C. Let $f$ be $\Omega$-stable and satisfy Axiom A, and let $\Lambda$ be a basic set of $\Omega(f)$. Then $W^{s}(\Lambda) \cap W^{u}(\Lambda)=\Lambda$, where $W^{s}(\Lambda)\left(W^{u}(\Lambda)\right)$ denotes the stable (unstable) manifold of $\Lambda$.

A modification of arguments in [5] gives the proof.

Proof of Proposition. Assume that $f$ and $g$ satisfy Axiom $\mathrm{A}$ and No cycle property. Generally it holds that $\Omega(f \times g) \subset \Omega(f) \times \Omega(g)$ and $\operatorname{Per}(f \times g)=$ $\operatorname{Per}(f) \times \operatorname{Per}(g)$. Since the periodic points of $f$ and $g$ are dense in their nonwandering sets, we have $\Omega(f \times g)=\Omega(f) \times \Omega(g)$. From this, it is clear that $f \times g$ satisfies Axiom A. Let us prove that $f \times g$ has No cycle property. Let

$$
\Omega(f)=\Omega_{1} \cup \cdots \cup \Omega_{l}, \quad \Omega(g)=\Lambda_{1} \cup \cdots \cup \Lambda_{m}, \quad \Omega(f \times g)=\Gamma_{1} \cup \cdots \cup \Gamma_{n}
$$

be decompositions to their basic sets. Suppose that $f \times g$ has a cycle. With no loss of generality, we can assume that $\Gamma_{1} \geqslant \Gamma_{2} \geqslant \cdots \geqslant \Gamma_{r} \geqslant \Gamma_{1}$ where $r \geqslant 2, \Gamma_{i} \neq \Gamma_{i+1}$ for all $1 \leqslant i \leqslant r-1$ and $\Gamma_{r} \neq \Gamma_{1}\left(\Gamma_{i} \geqslant \Gamma_{j}\right.$ means $\left.W^{u}\left(\Gamma_{i}\right) \cap W^{s}\left(\Gamma_{j}\right) \neq \varnothing\right)$. Since any $\Gamma_{i}$ is contained in some $\Omega_{j} \times \Lambda_{k}$, let $\Gamma_{i} \subset \Omega_{s_{i}} \times \Lambda_{t_{i}}$ for all $1 \leqslant i \leqslant r$. Clearly,

$$
W^{s}\left(\Gamma_{i}\right) \subset W^{s}\left(\Omega_{s_{i}}\right) \times W^{s}\left(\Lambda_{t_{i}}\right), \quad W^{u}\left(\Gamma_{i}\right) \subset W^{u}\left(\Omega_{s_{i}}\right) \times W^{u}\left(\Lambda_{t_{i}}\right) .
$$

Therefore it holds that $W^{u}\left(\Omega_{s_{i}}\right) \cap W^{s}\left(\Omega_{s_{i+1}}\right) \neq \varnothing, W^{u}\left(\Lambda_{t_{i}}\right) \cap W^{s}\left(\Lambda_{t_{i+1}}\right) \neq \varnothing$ for all $1 \leqslant i \leqslant r-1$, and $W^{u}\left(\Omega_{s_{r}}\right) \cap W^{s}\left(\Omega_{s_{1}}\right) \neq \varnothing, W^{u}\left(\Lambda_{t_{r}}\right) \cap W^{s}\left(\Lambda_{t_{1}}\right) \neq \varnothing$. Thus, we get $\Omega_{s_{1}} \geqslant \Omega_{s_{2}} \geqslant \cdots \geqslant \Omega_{s_{r}} \geqslant \Omega_{s_{1}}$ and $\Lambda_{t_{1}} \geqslant \Lambda_{t_{2}} \geqslant \cdots \geqslant \Lambda_{t_{r}} \geqslant \Lambda_{t_{1}}$. Since $f$ and $g$ have No cycle property, we have $\Omega_{s_{1}}=\Omega_{s_{j}}$ and $\Lambda_{t_{1}}=\Lambda_{t_{j}}$ for all $1 \leqslant j \leqslant r$. From this fact, $(*)$ and Lemma $C$,

$$
W^{u}\left(\Gamma_{1}\right) \cap W^{s}\left(\Gamma_{2}\right) \subset \Omega(f \times g) .
$$

This is a contradiction because $\Gamma_{1} \neq \Gamma_{2}$.

\section{REFERENCES}

1. S. D. Liao, On the stability conjecture, Chinese Ann. Math. 1 (1980), 9-29.

2. R. Mañé, Expansive diffeomorphisms, Dynamical Systems-Warwick, Lecture Notes in Math., vol. 468, Springer-Verlag, Berlin and New York, 1974.

3. , Contributions to the stability conjecture, Topology 17 (1978), 383-396.

4. Z. Nitecki, Differentiable dynamics, MIT Press, 1971. 
5. J. Palis, A note on $\Omega$-stability, Global Analysis, Proc. Sympos. Pure Math., vol. 14, Amer. Math. Soc., Providence, R.I., 1970, pp. 221-222.

6. J. Palis and S. Smale, Structural stability theorems, Global Analysis, Proc. Sympos. Pure Math., vol. 14, Amer. Math. Soc., Providence, R.I., 1970, pp. 223-231.

7. V. A. Pliss, $A$ hypothesis due to Smale, Differential Equations 8 (1972), 203-214.

8. _ The location of separatrices of periodic saddle-point motion of systems of second-order differential equations, Differential Equations 7 (1971), 906-927.

9. J. Robbin, A structural stability theorem, Ann. of Math. (2) 94 (1971), 447-493.

10. C. Robinson, $C^{r}$ structural stability implies Kupka-Smale, Dynamical Systems (M. Peixoto, ed.), Academic Press, 1973.

11. __ Structural stability of $C^{\prime}$ diffeomorphisms, J. Differential Equations 22 (1976), 28-73.

12. A. Sannami, The stability theorems for discrete dynamical systems on two-dimensional manifolds, Nagoya Math. J. 90 (1983) (to appear). (Announcement and summary: Proc. Japan Acad. Ser. A Math. Sci. 57 (1981), 403-407.)

13. S. Smale, The $\Omega$-stability theorem, Global Analysis, Proc. Sympos. Pure Math., vol. 14, Amer. Math. Soc. Providence, R.I., 1970, pp. 289-297.

Department of Mathematics, Hokkaido University, Sapporo, Japan 